
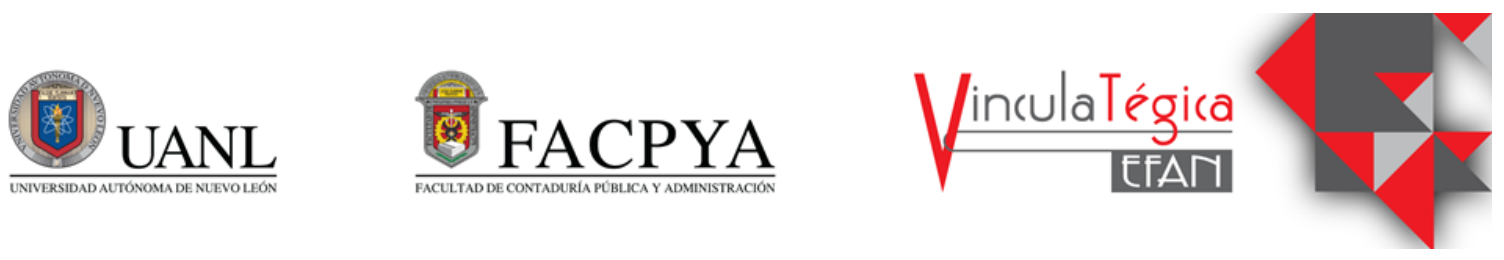

\title{
Diseño y validación de un instrumento que permita identificar los factores que influyen en la productividad laboral
}

\author{
Joel Meraz Sepúlveda ${ }^{1}$, Mayda González Espinoza ${ }^{2}$ y Gloria Muñoz del Real ${ }^{3}$ \\ ${ }^{1}$ Universidad Autónoma de Baja California, Facultad de Ciencias Administrativas Mexicali, Baja California, \\ México, meraz.joel@uabc.edu.mx, Calzada de los presidentes y Eje central, Río Nuevo, 21120 Mexicali, B.C., \\ (+52) 6862120809 \\ ${ }^{2}$ Universidad Autónoma de Baja California, Facultad de Ciencias Administrativas Mexicali, Baja California, \\ México,mayda.gonzalez@uabc.edu.mx Calzada de los presidentes y Eje central, Río Nuevo, 21120 Mexicali, B.C., \\ (+52) 6869453461 \\ ${ }^{3}$ Universidad Autónoma de Baja California, Facultad de Ciencias Administrativas Mexicali, Baja California, \\ México, gloria.munoz@uabc.edu.mx, Calzada de los presidentes y Eje central, Río Nuevo, 21120 Mexicali, B.C.,
} (+52) 6861199350

\author{
Información del artículo revisado por pares \\ Fecha de aceptación: junio-2021 \\ Fecha de publicación en línea: diciembre-2021 \\ DOI: https://doi.org/10.29105/vtga7.1-122
}

\section{Resumen}

Uno de los objetivos de toda organización sea pública o privada es maximizar la productividad de sus trabajadores para poder ser más eficientes y eficaces al brindar los productos y servicios que ofrecen. En este sentido uno de los grandes problemas de los sistemas de justicia es la falta de presupuesto para hacerle frente a las necesidades de mejora en las diversas áreas de la organización. Es así como el objetivo de este trabajo se centró en diseñar y validar un instrumento que permitiera detectar aquellos factores o variables que influyen en la productividad laboral de los notificadores del Centro de Justicia Penal unidad Río Nuevo, en la ciudad de Mexicali, Baja California; y así, poder enfocar el recurso económico en un área que provoque un mayor impacto. El instrumento diseñado consistió en un cuestionario de 69 ítems que se validó mediante diversas pruebas estadísticas: entre ellas el alfa de Cronbach, KMO, esfericidad de Bartlett y comunalidades. Se demostró que de entre las variables analizadas existe mayor correlación entre la productividad laboral y capacitación, quedando en evidencia que existe asociación entre las variables analizadas. Es así, que el instrumento validado podrá ser de utilidad para analizar la productividad laboral en diversos campos de estudio debido a su construcción de carácter general.

Palabras clave: productividad laboral, capacitación, condiciones laborales, motivación, cargas de trabajo.

Códigos JEL: M10, M11, M12

Abstract

One of the objectives of any organization, whether public or private, is to maximize the productivity of its workers in order to be more efficient and effective in providing the products and services they offer. In this sense, one of the great problems of the justice systems is the lack of budget to face the needs for improvement in the various areas of the organization. Thus, the objective of this work focused on designing and validating an instrument that would allow detecting those factors or variables that influence the labor productivity of the notifiers of the Río Nuevo Unit Criminal Justice Center, in the city of Mexicali, Baja California; and thus, to be able to focus the economic resource in an area that causes a greater impact. The designed instrument consisted of a 69 -item questionnaire that was validated using various statistical tests: among them Cronbach's alpha, KMO, Bartlett's sphericity, and communalities. It was shown that among the variables analyzed there is a greater correlation between labor productivity and training, making it 
clear that there is an association between the variables analyzed. Thus, the validated instrument may be useful to analyze labor productivity in various fields of study due to its general construction.

Key Words: labor productivity, training, working conditions, motivation, workloads.

Jel Codes: M10, M11, M12

\section{INTRODUCCIÓN}

\section{Planteamiento del problema}

Los conflictos de carácter penal entre justiciables se llevan a cabo mediante audiencias dentro de los tribunales de justicia penal. Para llevar a cabo el desarrollo de estos procesos se cuenta con una administración judicial que da soporte a las tareas jurisdiccionales. Estas funciones administrativas son realizadas por un recurso humano, y al realizarse indebidamente provocan demoras en el proceso de impartición de justicia. Por este motivo se desea mejorar la eficiencia y eficacia del personal de notificaciones desarrollando un instrumento de medición que permita el análisis de los factores que influyen en la productividad de las funciones de los notificadores.

La productividad laboral está determinada por diversas variables, Marvel et al., (2011, p. 588) identifica como factores humanos que contribuyen a la productividad: la motivación, la satisfacción laboral, la participación, el aprendizaje y la formación, las competencias, el trabajo en equipo, el estrés y el burn out, el clima organizacional, el liderazgo y estilo gerencial, la cultura organizacional, la comunicación, los hábitos de trabajo, las actitudes y sentimientos, la toma decisiones, la solución de conflictos, la ergonomía, el compromiso, entre otras. Estos son factores psicológicos que afectan la productividad del individuo según la teoría de las relaciones humanas, las cuales tiene como principales representantes a Elton Mayo, John Dewey y Kurt Lewin.

Surge la necesidad de crear un instrumento con el que se puedan analizar las variables de capacitación, condiciones laborales, motivación, cargas de trabajo y su influencia en la productividad laboral debido a la escasez presupuestaria para abordar todos los problemas asociados a la justicia (Palma, 2017) y en consecuencia es necesario enfocar esfuerzos en un área específica que produzca mayor impacto. Hidalgo, (2006) hace mención que para el análisis de datos dentro de la investigación de las ciencias sociales es necesario crear instrumentos fiables y válidos. Por consiguiente, el objetivo de este trabajo es elaborar un instrumento que posea fiabilidad y validez para el análisis de los factores que influyen en la productividad de los trabajadores que realizan las notificaciones de las resoluciones judiciales ante partes implicadas en algún proceso.

\section{Pregunta de investigación}

¿El diseño y validación de un instrumento de medición permitirá la identificación de los factores que influyen mayormente en la productividad de los trabajadores que realizan las notificaciones de las resoluciones judiciales?

\section{Hipótesis}

La validación de un instrumento permitirá el análisis de los factores que ejercen influencia sobre la productividad laboral.

\section{Objetivo General}

Diseñar y validar un instrumento que permita determinar la influencia que la productividad laboral recibe sobre los factores de capacitación, motivación, condiciones laborales y cargas de trabajo. 


\section{MARCO TEÓRICO}

En este apartado desarrollarán las teorías propuestas por diversos autores que explican la variable de productividad laboral y sus variables relacionadas que son: capacitación, condiciones laborales, motivación y cargas de trabajo. El propósito de este apartado es fundamentar la estructura utilizada para la formar la operacionalización de las variables debido a la necesidad de generar un instrumento de medición en la investigación científica-social como lo mencionan Hidalgo (2006) y López et al. (2019)

\section{Productividad laboral}

Jaimes et al. (2018) desde un enfoque administrativo la productividad laboral (también mencionada como productividad del factor humano) es clave para evaluar y mejorar el desempeño y así cumplir uno de los objetivos de la organización. Está determinada por diversas variables, entre ellas la motivación, satisfacción, competencias, trabajo en equipo, formación y desarrollo, clima organizacional, entre otras.

Gutiérrez (2010) afirma que "incrementar la productividad es lograr mejores resultados considerando los recursos empleados para generarlos" (p. 21). Porter (1985) menciona que el capital humano puede mejorar la productividad. Ochoa (2014) añade que la "productividad es la eficiencia y eficacia que tiene el colaborador al realizar su trabajo" (p.1). Yonclei (2018) también menciona al respecto que la productividad laboral "es la mejora continua del logro colectivo, la consecuencia de la eficiencia con que se administra el talento humano en su conjunto" (p. 34). Se entiende que la eficiencia y eficacia están íntimamente relacionadas con la productividad, siendo parte integral de esta. Es así, que se pueden entender como dimensiones que conforman el concepto de productividad laboral.

Maella (2010) dice que entre mayor capacidad de una persona mayor productividad existirá, además, esta capacidad siempre se puede incrementar. Por su parte Velázquez (2016) menciona que las condiciones laborales son un factor que afecta la productividad laboral y el funcionamiento de una organización (párr. 13). En Morales y Masis (2014) se menciona que la productividad depende de la motivación y el lugar de trabajo para aumentar la eficiencia y eficacia del personal (p.45). También Marvel et al. (2011) al respecto opina que las cargas de trabajo son un aspecto físico y psicológico que afecta el comportamiento de las personas y estas afectan la productividad del individuo. (p. 557). Debido a lo anterior, son variables que influyen en la productividad laboral: capacitación, condiciones laborales, motivación y cargas de trabajo.

\section{Eficiencia y eficacia}

Fuentes (2012) define como dimensiones de la productividad a la eficiencia, efectividad y eficacia; la eficiencia solo toma en cuenta al recurso en cuanto a cantidad y no calidad; la efectividad relaciona el logro de objetivos con los que se proponen primeramente sin tomar en cuenta a qué costo; mientras que la eficacia se da en relación de la satisfacción del cliente, por lo que se vincula forzosamente con el concepto de calidad (p. 32-33). Calvo et al. (2018) mencionan que la eficiencia es el mejor uso de los recursos disponibles, o lo que es igual, lograr hacer más productos con menos insumos, por lo que está asociado con la productividad.

Para dejar claras las diferencias entre eficiencia y eficacia, Chiavenato afirma que "la eficiencia no se preocupa de los fines, sino sólo de los medios. El alcance de los objetivos previstos no entra en la esfera de competencia de la eficiencia; este es un asunto relacionado con la eficacia" (2011, p. 288).

\section{Capacitación}


Álvarez et al. define a la capacitación como "aquellas acciones que instruyen y preparan a una persona para la actividad laboral" (2017, p.9). Se entiende que, si se capacita a los individuos, podrán aumentar dicha capacidad y por lo tanto mejorarán su productividad. Maella define a la capacitación como "aquellos conocimientos y habilidades que una persona posee" $(2010$, p.3), entonces, la capacitación está en función de aumentar dichos conocimientos y habilidades. Medina (2014) menciona que se entiende por capacitación:

... al conjunto de procesos organizados, relativos tanto a la educación no formal como a la informal, dirigidos a prolongar y a complementar la educación inicial mediante la generación de conocimientos, el desarrollo de habilidades y el cambio de actitudes, con el fin de incrementar la capacidad individual y colectiva para contribuir al cumplimiento de la misión institucional, a la mejor prestación de servicios a la comunidad, al eficaz desempeño del cargo y al desarrollo personal integral (p. 24).

Entre mayor capacidad mayor productividad existirá, además, esta capacidad siempre se puede incrementar, por tanto, la capacitación está en función de aumentar conocimientos y habilidades. Taboada (2019) ofrece la siguiente definición sobre capacitación:

... un proceso continuo que debe ser planteado en base a las necesidades reales de cada empresa $\mathrm{u}$ organización, sirviendo esta para orientar al desarrollo de habilidades y conocimientos que ayuden al trabajador en su vida laboral que lleva día con día, siendo su desempeño cada vez mejor y brindando la posibilidad de generar una trayectoria duradera en la empresa que participa (p. 8).

Para este trabajo son considerados como dimensiones de capacitación a los conocimientos y habilidades mencionados por Maella (2010), Medina (2014) y Taboada (2019).

El conocimiento comprende la información relacionada con el cargo o puesto de trabajo (Ortega, 2017). En este sentido, Hellriegel (2011, citado en Ortega, 2017) amplía el aporte mediante lo siguiente:

La administración del conocimiento se refiere a la creación, protección, desarrollo y distribución de información y activos intelectuales. En la nueva economía, el capital humano tendrá más peso, porque las personas son las que crean y comparten el conocimiento. Los trabajadores del conocimiento de muchas organizaciones se han posicionado como emprendedores independientes (p. 12).

El diccionario de la Real Academia Española define habilidad como la "capacidad y disposición para algo"; o bien como, "cada una de las cosas que una persona ejecuta con gracia y destreza"; también es "cuando alguien utiliza sus mañas y destrezas con el objeto de conseguir algo" (2021, s/p). Para Álvarez, es el dominio de las técnicas (1990); Petrovski (1980) la definió como el dominio de actividades psíquicas y prácticas complejas y que es necesario para poder llevar a cabo de manera adecuada las actividades, conocimientos y habilidades de alguien; por su parte Savin (1976) la define como la capacidad del hombre para realizar una actividad mediante el desarrollo de la experiencia previa.

Brito (1984) ofrece una clasificación de las habilidades y las divide en dos categorías: según el plano en que transcurren; en habilidades prácticas o motrices y habilidades teóricas o intelectuales. Y según el contenido que asumen; habilidades deportivas, profesionales, laborales y docentes (p. 51). Utilizando la clasificación de Brito, se consideran como habilidades aquellas que son necesarias en el contexto del ámbito laboral sujeto de estudio. De esta forma y de acuerdo con el manual de organización y procedimientos del nuevo sistema de justicia penal, las habilidades necesarias por un notificador son: iniciativa, conocimiento organizativo, trabajo bajo presión, conocimiento y experiencia, actitud asertiva, manejo de información, 
autocontrol, comunicación, compromiso con la organización, búsqueda de información y preocupación por el orden y la calidad (Poder Judicial del Estado de Baja California, 2006, s/p).

\section{Condiciones laborales}

Velázquez menciona que entre las principales condiciones laborales que afectan a la productividad se encuentran el clima organizacional, las herramientas de trabajo y los espacios otorgados para ejercer las actividades (2016, párr. 3). Por tanto, se toman como dimensiones de las condiciones laborales al clima organizacional y las condiciones físicas que consideran a las herramientas y espacios de trabajo.

El clima organizacional según Chiavenato (2011, p. 49) se refiere al ambiente interno de la organización y cuando es alto se puede distinguir por relaciones de satisfacción, ánimo, interés y colaboración. Velázquez (2007) menciona lo siguiente: "si existe tensión entre los miembros del personal, los líderes no prestan atención a sus peticiones y a cambio exigen más de lo establecido, el trabajar bajo colaboración será imposible, provocando que las actividades se realicen de forma ineficiente" (párr. 14). Es así que se contemplan como indicadores del clima organizacional la colaboración, el interés, el ánimo y la satisfacción por parte de los trabajadores.

Elton Mayo, en una de sus investigaciones concluyó que, los ambientes, la iluminación, la reducción de ruidos, herramientas y equipos para los empleados eran elementos sustanciales [condiciones físicas], y que toda organización debe brindar a sus trabajadores para que sean más productivos (Pilligua et al., 2019). Por lo que se retoman para este trabajo mediante dos indicadores: espacios adecuados y herramientas-materiales necesarios.

\section{Motivación}

Hellriegel \& Slocum (2004, p. 117) concibe la motivación como "fuerzas que actúan sobre una persona o en su interior y provocan que se comporte de una forma específica, encaminada hacia una meta". Robbins, (2004) define a la motivación como "procesos que dan cuenta de la intensidad, dirección y persistencia del esfuerzo de un individuo por conseguir una meta" (p.155). Gray (2008, citado en Ochoa, 2014) explica que la motivación está constituida por todos los factores capaces de provocar, mantener y dirigir la conducta hacia un objetivo (p. 8). Por su lado, Porret (2008, citado en Ochoa, 2014) define motivación a lo que impulsa, dirige y mantiene el comportamiento humano dentro de la organización (p. 4).

Pizarro (2017) utiliza como dimensiones de la motivación a los dos factores de la teoría de Herzberg, la cual hace mención de que existen factores higiénicos y factores motivacionales que afectan el grado de satisfacción del individuo (p. 38). Se retomaron para este trabajo las dimensiones de Herzberg utilizadas por Pizarro.

Los factores higiénicos son aquellos factores que se encuentran en el entorno del individuo en su ambiente de trabajo, aun cuando se encuentren en un nivel óptimo solo evitan que el individuo se encuentre insatisfecho y por consiguiente no tienen un fuerte impacto en su motivación (Chiavenato, 2011, p. 45). También se les llaman factores de insatisfacción y son utilizados para los fines de este trabajo. Chiavenato menciona a: condiciones de bienestar, políticas de la organización y administración, relaciones con el supervisor, competencia técnica del supervisor, salario y remuneración, seguridad en el puesto y relaciones con los colegas.

Los factores motivacionales son aquellos que están relacionados directamente con los sentimientos del individuo hacia el puesto de trabajo y las funciones que desempeña. Si el individuo se encuentra cómodo con lo que hace se elevará su nivel de satisfacción ya que están relacionados con los niveles más altos de la 
pirámide de la jerarquía de las necesidades propuesto por Maslow. También llamados factores de satisfacción han sido utilizados como conceptos para la realización de los ítems en este trabajo; Chiavenato menciona los siguientes: delegación de responsabilidad, libertad para decidir cómo realizar una labor, posibilidades de ascenso, utilización plena de las habilidades personales, formulación de objetivos y evaluación relacionada con ellos, simplificación del puesto, ampliación o enriquecimiento del puesto (2011, p. 45).

\section{Cargas de trabajo}

Interactive Training Advanced Computer Applications [ITACA] define a la carga de trabajo como "el conjunto de exigencias físicas [carga física de trabajo] y exigencias psicológicas [carga psíquica de trabajo], que inciden sobre la persona en el desarrollo de su puesto de trabajo" (Rivera, 2017, p. 3). Esto se refiere al esfuerzo físico y mental que se ejerce dentro del trabajo para realizar diversas actividades, y como menciona Rivera, que se relaciona directamente con el rendimiento. Chavarría (1984) define a las cargas de trabajo como "el conjunto de requerimientos psicofísicos a los que el trabajador se ve sometido a lo largo de la jornada laboral" (p. 1). Arcos (2017) menciona que la carga de trabajo "genera presiones y conflictos que incrementan los niveles de estrés y por ende un deterioro en la salud física y psicológica" (p. 14). Toda carga de trabajo producida por una actividad que responde a las exigencias de una tarea presenta un componente de carga física y carga mental (Herrera, 2011, diapositiva 5).

Para efectos de este estudio se utilizarán las cargas físicas y las cargas psicológicas o mentales como dimensiones de cargas de trabajo [también llamadas cargas laborales] mencionadas por Rivera (2017) Chavarría (1984) y Arcos (2017).

La sobrecarga de trabajo tiene relación con el volumen, la extensión o dificultad de la tarea, es superior o inferior a la capacidad del sujeto para su desarrollo (Rivera, 2017, p. 11). Es pues, exceder la capacidad de carga de trabajo, que involucra componentes físicos y mentales. De lo anterior se genera el concepto de "fatiga física" entendida como la disminución de la capacidad física del individuo después de haber realizado un trabajo, durante un tiempo determinado. La fatiga de un individuo se puede hacer notoria cuando se incrementan errores como consecuencia de la realización de movimientos corporales más lentos, una disminución de la capacidad de coordinación de los mismos y mayor lentitud de los reflejos, aparición de una sensación de malestar e insatisfacción y disminución de la capacidad de trabajo, lo que produce una disminución del rendimiento del trabajador.

\section{MÉTODO}

Esta investigación parte de la revisión de la literatura en tesis, artículos, libros y revistas científicas de temas relacionados a la productividad del recurso humano desde un enfoque humanístico. Se toma como referencia la teoría de las relaciones humanas de Elton Mayo, en la cual, factores psicológicos son determinantes de la productividad de los trabajadores. Fue necesario elaborar la operacionalización de la variable para diseñar un instrumento de medición que evalúe la relación de las variables independientes con la productividad laboral. Se eligieron como variables que afectan a la productividad laboral; la capacitación, mencionada en Maella (2010) y Álvarez et al. (2017); las condiciones laborales, mencionadas en (Pilligua et al. (2019); la motivación, mencionada en (Morales et al. (2014) y Marvel et al. (2011); y cargas de trabajo, utilizadas por el mismo Marvel et al. (2011).

El instrumento constó de un cuestionario estructurado (Muñoz, 2020) con 10 ítems de control y 69 ítems escala tipo Likert con 5 posibles respuestas [Totalmente de acuerdo; De acuerdo; Ni de acuerdo ni en desacuerdo; En desacuerdo; Totalmente en desacuerdo] mencionadas en Francés et al. (2014, p. 139). 7 ítems integraron la variable dependiente productividad laboral; 16 ítems la variable capacitación; 22 ítems 
la variable condiciones laborales; 10 ítems la variable motivación; y 14 ítems la variable cargas de trabajo; cada una de las variables se integra a su vez por dos dimensiones (ver Tabla 1).

Tabla 1. Operacionalización de las variables

\begin{tabular}{|c|c|c|c|}
\hline Variables & Dimensión & Preguntas & Ítems \\
\hline Preguntas de control & & & 10 \\
\hline \multirow[t]{2}{*}{ Productividad laboral } & Eficiencia & P 1-3 & 3 \\
\hline & Eficacia & P 4-7 & 4 \\
\hline \multirow[t]{2}{*}{ Capacitación } & Conocimientos & Cap 1-7 & 7 \\
\hline & Habilidades & Cap 8-16 & 9 \\
\hline \multirow[t]{2}{*}{ Condiciones laborales } & Clima organizacional & CondL 1-13 & 13 \\
\hline & Condiciones físicas & CondL 14-22 & 9 \\
\hline \multirow[t]{2}{*}{ Motivación } & Factores higiénicos & M 1-6 & 6 \\
\hline & Factores motivacionales & M 7-11 & 4 \\
\hline \multirow[t]{2}{*}{ Cargas de trabajo } & Cargas físicas & CargT 1-5 & 5 \\
\hline & Cargas mentales & CargT 6-14 & 9 \\
\hline
\end{tabular}

Para determinar la validez y confiabilidad del instrumento, fue necesario realizar una prueba piloto misma que se aplicó a la población objetivo, conformada por nueve trabajadores del área de notificaciones del Centro de Justicia Penal unidad Río Nuevo en Mexicali Baja California. Debido a la situación de pandemia por el COVID-19, el cuestionario se hizo llegar vía electrónica, no sin antes haberles explicado la finalidad, los detalles y la importancia de expresar su percepción al contestar el instrumento.

La fiabilidad hace referencia a obtener los mismos resultados en diferentes pruebas aplicadas, la validez refiere a medir lo que se debe medir (Francés et al., 2014, p.87; Muñoz, 2020, p. 204). En otras palabras, la validez y confiabilidad otorgan exactitud y consistencia necesaria para efectuar generalizaciones mediante pruebas más rigurosas (Hidalgo, 2006). Por tanto, se procedió a realizar el análisis estadístico mediante el programa SPSS versión 25. Se realizaron como pruebas de fiabilidad el alfa de Cronbach, Kolmogorov-Smirnov y análisis de correlaciones mediante el coeficiente de Pearson. Para el análisis de validez del instrumento se realizaron las pruebas de Kaiser-Meyer-Olkin (KMO), Esfericidad de Bartlett y Comunalidades.

\section{RESULTADOS}

El coeficiente alfa de Cronbach es una fórmula general para estimar la fiabilidad de un instrumento en el que la respuesta tiene más de dos valores (Aiken, 2003). (Barrios y Cosculluela (2013) establece un margen de fiabilidad para el alfa de Cronbach con valores entre 0,70 y 0,95 , y que un resultado perfecto de 1 proporciona datos redundantes o irrelevantes (p. 11). Mientras que Nunnally (1978) señala que la puntuación mínima aceptable puede ser de $\geq 0.70$. El resultado obtenido para el alfa de Cronbach en el total de las variables que integran el instrumento fue de 0.950 (ver tabla 2). Por tanto, se afirma que el instrumento es fiable y que se obtendrán los mismos resultados en diversas pruebas. 
Tabla 2. Alfa de Cronbach

$\frac{\text { Alfa de Cronbach }}{0.950}$

La prueba de Kolmogorov-Smirnov o prueba K-S permite comparar dos distribuciones de probabilidad entre sí (Diego, 2018, p. 10) y se puede definir el tipo de correlación a utilizar de acuerdo con el valor de significancia resultante. Si se obtienen valores $>0.05$ quiere decir que existe una distribución normal de las variables, por lo que se debe utilizar Pearson. Un resultado < 0.05 significa que no hay una distribución normal de las variables por lo que debe usar la prueba no paramétrica de Spearman. Las variables productividad laboral, condiciones laborales, motivación y cargas de trabajo obtuvieron valores superiores a 0.05; solo la variable capacitación obtuvo un valor menor 0.05 (ver tabla 3). Por lo que se afirma que la distribución de las variables es normal y, por tanto, se utilizó la prueba de Pearson para el análisis paramétrico de correlaciones.

Tabla 3. Prueba Kolmogorov-Smirnov

\begin{tabular}{ccccc}
\hline $\begin{array}{c}\text { productividad } \\
\text { laboral }\end{array}$ & capacitación & $\begin{array}{c}\text { condiciones } \\
\text { laborales }\end{array}$ & motivación & $\begin{array}{c}\text { cargas de } \\
\text { trabajo }\end{array}$ \\
\hline 0.200 & 0.007 & 0.200 & 0.200 & 0.200 \\
\hline Elaboración propia.
\end{tabular}

Restrepo y González (2007) afirma que "Un coeficiente de correlación mide el grado de relación o asociación existente generalmente entre dos variables aleatorias" (p. 187). Para el análisis de correlación de pruebas paramétricas y no paramétricas existen diversas pruebas, entre ellas el coeficiente de Pearson, el cual es "una estadística inferencial que refleja la intensidad de la asociación lineal entre dos variables cuantitativas" (Manterola et al., 2018, p, 682). Tiene como objetivo medir la fuerza o grado de asociación entre dos variables aleatorias cuantitativas que poseen una distribución normal bivariada conjunta (Restrepo et al., 2007, p. 185).

Al realizar la prueba para el análisis de correlaciones mediante Pearson, en los resultados se aprecia una fuerte correlación entre las variables capacitación-productividad laboral de 0.861 y significancia de 0.003 . Las variables cargas de trabajo-productividad laboral presentan un coeficiente de 0.631 , sin embargo, la correlación no es significante debido a que presenta un valor de 0.068 . Las variables condiciones laboralescargas de trabajo presentan una correlación de 0.767 y significancia de 0.016 . Mientras que las variables motivación-cargas de trabajo resultan con una correlación de 0.727 y valor de significancia de 0.026 (ver tabla 4). Por tanto, existe correlación positiva y significante en al menos una variable dependiente con productividad laboral, y existe asociación entre las mismas variables dependientes.

Tabla 4. Matriz de correlaciones

\begin{tabular}{|c|c|c|c|c|c|}
\hline & $\begin{array}{l}\text { Productividad } \\
\text { laboral }\end{array}$ & Capacitación & $\begin{array}{c}\text { Condiciones } \\
\text { laborales }\end{array}$ & Motivación & Cargas de trabajo \\
\hline $\begin{array}{l}\text { Productividad } \\
\text { laboral }\end{array}$ & 1 & & & & \\
\hline
\end{tabular}




\begin{tabular}{|c|c|c|c|c|c|}
\hline Capacitación & 0.861 & 1 & & & \\
\hline Sig. & 0.003 & & & & \\
\hline $\begin{array}{l}\text { Condiciones } \\
\text { laborales }\end{array}$ & 0.475 & 0.528 & 1 & & \\
\hline Sig. & 0.197 & 0.144 & & & \\
\hline Motivación & 0.446 & 0.195 & 0.610 & 1 & \\
\hline Sig. & 0.229 & 0.614 & 0.081 & & \\
\hline Cargas de trabajo & 0.631 & 0.588 & 0.767 & 0.727 & 1 \\
\hline Sig. & 0.068 & 0.096 & 0.016 & 0.026 & \\
\hline
\end{tabular}

Elaboración propia.

La prueba de adecuación de Kaiser-Meyer Olkin (KMO) "permite valorar el grado en que cada una de las variables es predecible a partir de las demás" (López-Aguado y Guitiérrez-Provecho, 2019, p. 7). Los autores como Ferguson y Cox (1993); y (Hair et al. (2005), toman un valor de 0.50 como punto de corte mínimo aceptable; y otros como Costello Osborne (2005); y Ferrando Anguiano-Carrasco (2010), toman un valor mínimo de 0.70 o incluso 0.80 . El instrumento obtuvo un KMO de 0.586 (ver tabla 5), por lo que el resultado se considera aceptable retomando el punto de corte utilizado por (Ferguson y Cox (1993); y Hair et al. (2005).

Tabla 5. Prueba KaiserMeyer Olkin

$\frac{\mathrm{KMO}}{0.586}$

Por otra parte, la prueba de esfericidad de Bartlett "pone a prueba la hipótesis nula de que las variables analizadas no están correlacionadas en la muestra" (López-Aguado y Guitiérrez-Provecho, 2019, p. 6), en la cual su significación será mejor cuando se encuentre por debajo de 0.05. En la prueba de esfericidad de Bartlett se obtuvo una significancia de 0.009 (ver tabla. 6), por tanto, existe correlación entre las variables debido a que su significancia es $<0.05$. Por consiguiente, se afirma que las variables son explicadas por los valores comunes.

Tabla 6. Prueba esfericidad de Bartlett

\begin{tabular}{c}
\hline Bartlett \\
\hline 0.009 \\
\hline
\end{tabular}

Elaboración propia SPSS

Las comunalidades son unos valores que oscilan entre 0 y 1 . Cuando se aproxima a 1 indica que la variable queda totalmente explicada por los factores comunes; mientras que, si se aproxima a 0 , los factores no explicarán nada la variabilidad de las variables (Rodríguez-Jaume y Mora-Catalá, 2001). Al realizar el análisis de comunalidades, todas las variables obtuvieron un coeficiente por encima de 0.4 siendo la motivación la variable con el resultado más bajo de 0.521 (ver tabla 7). Quiere decir que la variabilidad de todas las variables sí esta explicada por los factores utilizados. 
Tabla 7. Extracción de Comunalidades y Matriz del Componente

\begin{tabular}{lcc}
\hline \multicolumn{1}{c}{ Variable } & Comunalidades & $\begin{array}{c}\text { Matriz del } \\
\text { componente }\end{array}$ \\
\hline Productividad laboral & 0.698 & 0.836 \\
Capacitación & 0.610 & 0.781 \\
Condiciones laborales & 0.688 & 0.830 \\
Motivación & 0.521 & 0.721 \\
Cargas de trabajo & 0.834 & 0.914 \\
\hline Elaboración propia & &
\end{tabular}

Para que el instrumento pasara las pruebas de fiabilidad y validez realizadas fue necesario ajustarlo, esto consistió en eliminar 23 de los 69 ítems iniciales, resultando 46 ítems finales: 6 para la variable productividad laboral, 16 para capacitación, 14 para condiciones laborales, 5 para motivación y 7 para cargas de trabajo (ver tabla 8).

Tabla 8. Resumen de pruebas de Fiabilidad y Validez del Instrumento

\begin{tabular}{ccccc}
\hline $\begin{array}{c}\text { Alfa de } \\
\text { Cronbach }\end{array}$ & KMO & Bartlett & Varianza & ítems \\
\hline 0.950 & 0.586 & 0.009 & $67.024 \%$ & 46 \\
\hline Elaboración propia del autor & & &
\end{tabular}

\section{CONCLUSIONES}

Mediante este estudio se confirma la hipótesis de que el instrumento diseñado es confiable y válido, por lo que es útil en su conjunto para analizar la correlación existente de variables que tienen influencia en la productividad laboral. El instrumento validado será utilizado en el desarrollo de la investigación que se encuentra en curso para la tesis de maestría realizada por el autor, y que trata del mismo tema abordado.

Los resultados del instrumento permiten detectar que, para el caso particular de la población encuestada, la capacitación del personal es el factor que mas influye en la productividad laboral. Presenta una correlación de 0.861 y una significancia de 0.003 , por lo que es una relación positiva y significante. Esto concuerda con los trabajos hechos por Palacios (2018) y Valentin (2017), entendiéndose que entre más y mejor capacitados estén los individuos, en cuanto habilidades y conocimientos relacionados con el trabajo, tendrán mayor productividad.

Debido a que el instrumento permite el análisis de las variables en estudio, abre una posibilidad de abordar problemas administrativos dentro de organizaciones judiciales desde una perspectiva diferente a la que se ha realizado hasta hoy. Existe poca información de estudios con enfoque administrativo dentro de organizaciones creadas para la impartición de justicia, y esto se puede adjudicar a la confusión que todavía existe entre las funciones administrativas y las jurisdiccionales. Se desea pues, que sea cada vez más frecuente la aplicación de instrumentos que tengan por finalidad coadyuvar a las funciones jurisdiccionales en el mejoramiento del proceso de impartición de justicia. 
Por lo ya mencionado, se vuelve aún más importante para el estudio de las ciencias sociales el aporte de instrumentos que proporcionen información válida que sustente las acciones emprendidas para mejorar cualquier organización, independientemente del sector al que pertenezcan, puesto que están conformadas por el recurso más valioso que es la gente. 


\section{REFERENCIAS}

Aiken, L. R. (2003). Tests psicológicos y evaluación. En Test Psicológicos y evaluación (11va ed.). Pearson Educación. https://ebg.ec/wp-content/uploads/2020/10/Tests-psicologicos-y-evaluacion-1.pdf

Álvarez, B., Freire, D., \& Gutiérrez, B. (2017). Capacitación y su impacto en la productividad laboral de las empresas chilenas [Universidad de Concepción]. http://repositorio.udec.cl/bitstream/11594/2495/3/Álvarez - Freire - Gutiérrez.pdf

Álvarez, R. M. (1990). El desarrollo de habilidades en la enseñanza de la historia. Editorial Pueblo y Educación.

Arcos, M. F. (2017). La sobrecarga de trabajo y su efecto sobre el compromiso organizacional en la gerencia de negocios de una empresa de telecomunicaciones [tesis de maestría, Universidad Andina Simón Bolivar]. http://repositorio.uasb.edu.ec/bitstream/10644/6057/1/T2551-MDTH-Arcos-La sobrecarga.pdf

Barrios, M., \& Cosculluela, A. (2013). Capitulo II Fiabilidad. En Psicometría (1a ed., pp. 75-140). Editorial UOC.

Brito, H. (1984). Hábitos, habilidades y capacidades. Revista Varona, 7(13).

Calvo, J., Pelegrín, A., \& Gil, M. S. (2018). Enfoques teóricos para la evaluación de la eficiencia y eficacia en el primer nivel de atención médica de los servicios de salud del sector público. Retos de la Dirección, 12(1), 96-118. http://scielo.sld.cu/pdf/rdir/v12n1/rdir06118.pdf

Chavarría, R. (1984). NTP 177: La carga física de trabajo: definición y evaluación. Centro Nacional de Condiciones de Trabajo - Barcelona. http://www.insht.es/InshtWeb/Contenidos/Documentacion/FichasTecnicas/NTP/Ficheros/101a200/n tp_177.pdf

Chiavenato, I. (2011). Administración de recursos humanos. El capital humano de las organizaciones (9a. ed). McGRAW-HILL/INTERAMERICANA EDITORES, S.A. DE C.V.

Costello, A. B., \& Osborne, J. W. (2005). Best practices in exploratory factor analysis: four recommendations for getting the most from your analysis. Practical Assessment, Research, and Evaluation, 10(7), 1-9. doi: https://doi.org/10.7275/jyj1-4868

Diego, A. (2018). Test de bondad de ajuste multivariable [grado en matemáticas, Universidad de Cantabria]. https://repositorio.unican.es/xmlui/bitstream/handle/10902/15639/Diego Gutierrez Andrea.pdf?sequence $=1 \&$ isAllowed $=\mathrm{y}$

Ferguson, E., \& Cox, T. (1993). Exploratory Factor Analysis: A Users'Guide. International Journal of Selection and Assessment, 1, 84-94. https://doi.org/https://doi.org/10.1111/j.14682389.1993.tb00092.x

Ferrando, P. J., \& Anguiano-Carrasco, C. (2010). El análisis factorial como técnica de investigación en psicología. Papeles del Psicólogo, $31(1), \quad 3-33$. http://www.redalyc.org/articulo.oa?id=77812441003

Francés, F. J., Alaminos, A., Penalva, C., \& Santacreu, Ó. A. (2014). El proceso de medición de la realidad social: La investigación a través de encuestas. PYDLOS Ediciones. http://hdl.handle.net/10045/52605

Fuentes, S. M. (2012). Satisfacción laboral y su influencia en la productividad (Estudio realizado en la delegación de Recursos Humanos del Organismo Judicial en la ciudad de Quetzal Tenango. Universidad Rafael Landívar.

Gutiérrez, H. (2010). Calidad total y Productividad (3a ed.). McGRAW-HILL/INTERAMERICANA EDITORES, S.A. DE C.V. https://clea.edu.mx/biblioteca/files/original/56cf64337c2fcc05d6a9120694e36d82.pdf

Hair, J. F., Anderson, R. E., Tatham, R. L., \& Black, W. C. (2005). Análisis multivariante (6a ed.). Pearson Prentice Hall.

Hellriegel, D., \& Slocum, J. W. (2004). Comportamiento organizacional (10ma ed.). THOMSON.

Herrera, E. (2011). Cargas físicas del trabajo [presentación online]. slideshare. https://es.slideshare.net/osvaldoeltoch/carga-fisica-del-trabajo

Hidalgo, L. (2006). Confiabilidad y validez en el contexto de la investigación y evaluación cualitativa. 
Paradigma, 27, 07-33. http://www.ucv.ve/uploads/media/Hidalgo2005.pdf

Jaimes, L., Luzardo, M., \& Rojas, M. D. (2018). Factores Determinantes de la productividad laboral en pequeñas y medianas empresas de confecciones del área metropolitana de Bucaramanga, Colombia. Información tecnológica, 29(5), 175-186. https://doi.org/10.4067/s0718-07642018000500175

López-Aguado, M., \& Guitiérrez-Provecho, L. (2019). Cómo realizar e interpretar un análisis factorial exploratorio utilizando SPSS. Revista d'Innovació i Recerca en Educació REIRE, 12(2), 1-14. https://doi.org/10.1344/reire2019.12.227057

López, R., Avello, R., Palmero, D. E., Sánchez, S., \& Quintana, M. (2019). Validación de instrumentos como garantía de la credibilidad en las investigaciones científicas. Revista Cubana de Medicina Militar, 48(2). http://www.revmedmilitar.sld.cu/index.php/mil/article/view/390/331

Maella, P. (2010). Las variables y las conductas de la eficacia personal. IESE Occasional Paper. https://media.iese.edu/research/pdfs/OP-0174.pdf

Manterola, C., Grande, L., Otzen, T., García, N., Salazar, P., \& Quiroz, G. (2018). Confiabilidad, precisión o reproducibilidad de las mediciones. Métodos de valoración, utilidad y aplicaciones en la práctica clínica. Revista chilena de infectología, 35(6), 680-688. https://doi.org/10.4067/s071610182018000600680

Marvel, M., Rodríguez, C., \& Núñez, M. A. (2011). La productividad desde una perspectiva humana: $\begin{array}{llll}\text { Dimensiones } & \text { y } & \text { factores. } & \text { Intangible }\end{array}$ https://doi.org/10.3926/ic.2011.v7n2.p549-584

Medina, F. I. (2014). Diseño del plan de capacitación en base al modelo de retorno de inversión - ROIpara la planta central del ministerio de defensa nacional, correspondiente al periodo fiscal 2013 [tesis de maestría, Pontificia Universidad Católica del Ecuador]. http://repositorio.puce.edu.ec/handle/22000/8130

Morales, C., \& Masis, A. (2014). La medicion de la productividad del valor agregado: una aplicación empírica en una cooperativa agroalimentaria de Costa Rica. TEC Empresarial, 8(2), 41-49. https://doi.org/10.18845/te.v8i2.1988

Muñoz, G. (2020). Estudio de caso: metodología científica y su análisis estadístico en las ciencias sociales (1a ed.). EDICIONES ILCSA S.A. de C.V.

Nunnally, J. C. (1978). Psychometric theory (2nd ed.). McGraw-Hill.

Ochoa, K. A. C. (2014). Motivación y productividad laboral. Estudio realizado en la Empresa Municipal Aguas de Xelaju EMAX [Facultad de Humanidades, Universidad Rafael Landívar]. http://recursosbiblio.url.edu.gt/tesiseortiz/2014/05/43/Ochoa-Katleen.pdf

Ortega, R. A. (2017). Diseño organizacional y su efecto en la retención del capital humano: El caso de PYME familiar librería universitaria [tesis de maestría, Universidad Autónoma de Baja California]. https://drive.google.com/file/d/10SCtX_ofLJn9qvOhDctejIBYKnymqHvL/view\#

Palacios, J. C. (2018). La capacitación y su relación con la productividad laboral de los colaboradores del área de Marketing y promoción de la Universidad César Vallejo Chimbote, 2018. https://repositorio.ucv.edu.pe/bitstream/handle/20.500.12692/39388/Palacios_FJC.pdf?sequence=1 \&isAllowed $=\mathrm{y}$

Palma, L. M. (2017). Modernización Judicial, Gestión Y Administración En América Latina. Acta Sociológica, 72, 149-203. https://doi.org/10.1016/j.acso.2017.06.007

Petrovski, A. (1980). Psicología general. Editorial Progreso.

Pilligua, L., Fabricio, C., Ureta, A., \& María, F. (2019). El Clima organizacional como factor clave para el rendimiento productivo de las empresas. Estudio caso: Hardepex Cía. Ltda. Cuadernos $\begin{array}{llll}\text { Latinoamericanos Administración, } & \text { de }\end{array}$ http://www.redalyc.org/articulo.oa?id=409659500007

Pizarro, M. O. (2017). Motivación y productividad laboral en los colaboradores de la oficina de admisión de una institución privada, 2016 [tesis de maestría, Universidad César Vallejo]. https://hdl.handle.net/20.500.12692/8719

Poder Judicial del Estado de Baja California. (2006). Manual de organización y procedimientos. Nuevo Sistema de Justicia Penal Baja California. Tribunal de Garantía y Juicio Oral Penal. 
http://nsjp.pjbc.gob.mx:8088/SJPO/Resources/Documentos/manual_de_organizacion_y_procedimie ntos.pdf

Porter, M. (1985). Ventaja competitiva.

Real Academia Española. (2021). Habilidad. En Diccionario de la Lengua Española. https://dle.rae.es/habilidad?m=form

Restrepo, L. F., \& González, J. (2007). De Pearson a Spearman. Revista Colombiana de Ciencias Pecuarias, 20(2), 183-192. https://www.redalyc.org/pdf/2950/295023034010.pdf

Rivera, A. B. (2017). Efecto de la carga de trabajo en el desempeño de los trabajadores [tesis de grado, Universidad Militar Nueva Granada]. https://repository.unimilitar.edu.co/bitstream/handle/10654/16216/RiveraSanche?sequence=1

Robbins, S. P. (2004). Comportamiento organizacional (10ma ed.). Pearson Educación.

Rodríguez-Jaume, M.-J., \& Mora-Catalá, R. (2001). Análisis de regresión múltiple. Capítulo 8 Análisis factorial. Universidad de alicante. https://doi.org/10.3726/978-1-4539-1172-3/10

Savin, N. V. (1976). Pedagogía. Editorial Pueblo y Educación.

Taboada, L. Y. (2019). Impacto de la capacitación en el desempeño laboral en el área de atención al cliente en una empresa de renta y venta de copiadoras [tesis de maestría, Universidad Autónoma de Baja California]. https://drive.google.com/file/d/1CHtBZ7ClXD-hPTiCmDqeivsosBuFBpUP/view\#

Valentin, E. W. (2017). La capacitación y la productividad de los trabajadores del banco de crédito del perú los olivos. https://repositorio.ucv.edu.pe/bitstream/handle/20.500.12692/14036/Valentin_FEW.pdf?sequence=1 \&isAllowed=y

Velázquez, G. (2007). Las organizaciones y el capital humano. Revista Mundo Siglo XXI, 9, 81-90. http://www.mundosigloxxi.ciecas.ipn.mx/pdf/v03/09/05.pdf

Velázquez, M. (2016). 5 factores que afectan la productividad en el trabajo [artículo de revista online]. Entrepreneur. https://www.entrepreneur.com/article/280867

Yonclei, G. H. (2018). Satisfaccion laboral y la productividad de los trabajadores de la municipalidad provincial de Huaura, 2017 [Universidad Nacional Jose Faustino Sanchez Carrion]. http://repositorio.unjfsc.edu.pe/handle/UNJFSC/1870 